\title{
Pericardial effusion as a complication of myocardial infarction : How and what to do?
}

\author{
Bunga Novitalia ${ }^{\mathrm{a}, a}$, Muhammad Aminuddin ${ }^{a *}$ \\ abunga.novitalia-2017@fk.unair.ac.id \\ ${ }^{a}$ Department of Cardiology and Vascular Medicine, Faculty of Medicine, Airlangga University - Dr. Soetomo General Hospital, \\ Mayjen Prof. Dr. Moestopo 6-8, Surabaya 60286, Indonesia
}

\begin{abstract}
Pericardial effusion after myocardial infarction is relatively common, however the management and prognosis is not always straightforward. Regardless of effusion size, data suggests that pericardial effusion which occurs early post myocardial infarction were correlated with higher morbidity and mortality. This report describes a case of pericardial effusion occurrence after myocardial infarction and reviews published literature about the etiology and management of this complication. A 59 years old man with typical chest pain since 26 hours before refered to emergency room, sign and symptoms of cardiogenic shock, elevated cardiac marker, and electrocardiogram showing ST elevation in V1-V6, I, aVL (extensive anterior). He was observed and conservatively treated in intensive cardiology care unit. After 9 days of care, moderate pericardial effusion is revealed by echocardiography examination, which did not exist at the admission. Pericardial effusion (PE) as the complication of myocardial infarction is associated with anterior ST-segment elevation myocardial infarction (STEMI) and when there is heart failure. Poor ST-segment resolution is clearly associated with presence of PE and especially with tamponade or electromechanical dissociation. The most frequent cause of moderate PE in STEMI patients is bleeding from the infarcted wall. STEMI patients with moderate PE have increased mortality than those with small PE or without PE, and death was attributable to free wall rupture (FWR). It is necessary to detect PE earlier with echocardiography and some deserve close monitoring to detect FWR.
\end{abstract}

Keywords: pericardial effusion, myocardial infarction, free wall rupture

\section{Introduction}

Acute myocardial infarction (AMI) has a major contribution in human mortality(Hafiz-Ur-Rehman et al. 2010). Following an AMI, patients are at risk for a variety of cardiac complications. Pericardial effusion (PE) is not an uncommon finding in serial echocardiographic examination of patients with AMI as a complication(Mazzoni et al. 2000). With modern therapies including early reperfusion strategies, its incidence has decreased. In MI cases, PE is reported currently in 4\% to 19\%(Bière et al. 2015). PE in AMI is correlated with worse outcome. Circulatory failure from severe left ventricular (LV) dysfunction or from one of the complications of AMI involving PE cause in hospital morbidity among patients with AMI(Hafiz-Ur-Rehman et al. 2010).

The majority of PE does not cause hemodynamic instability, and a minimal to moderate size of PE may 
often be considered as a benign reaction to the myocardial infarction (MI)(Varma 2016). But moderate ( $\geq 10$ $\mathrm{mm}$ ) to severe PE in patients with ST-elevation MI (STEMI) should be considered a risk factor for acute or subacute LV free wall rupture (FWR), potentially causing tamponade which presents as electromechanical dissociation (EMD) or arterial hypotension, and sudden cardiac death(Bière et al. 2015; Figueras et al. 2010; Varma 2016). The mortality was twice higher in patients without moderate PE, even with no presence of EMD or cardiac tamponade(Figueras et al. 2010).

A study strongly recommended early screening to identify high-risk patients who need much closer monitoring. Obtained data that explains when PE is found early after MI could be a sign that FWR will emerge later(Køber, Møller, and Torp-Pedersen 2010). The aim of this case report is to present the detail of a patient with $\mathrm{PE}$ as a complication of AMI, describe the mechanism, and how to manage this complication.

\section{Case Description}

A 59-year-old Asian male presented to the emergency room with typical chest pain (the onset is 50 hours before), dyspnea, nausea, and cold sweating. He also had history of hypertension and dyslipidemia, without prior same complaints and familial history of heart disease. Initial vital signs were notable for heart rate of $128 \mathrm{x} /$ minute, blood pressure $60 / 30 \mathrm{mmHg}$, respiratory rate $30 \mathrm{x} /$ minute, and oxygen saturation of $91 \%$ on room air. Physical exam demonstrated basal bilateral rales and elevated jugular venous pressure. Initial laboratory values were remarkable for leukositosis, and significant increasing of cardiac marker. Presenting electrocardiogram (ECG) (Fig. 1) revealed sinus tachycardia with left axis deviation, counter clockwise rotation, complete right bundle branch block (RBBB) and ST elevation in V1-V6, I, aVL (extensive anterior). The chest $\mathrm{x}$-ray examination showed an increase in the bronchovascular patch indicating pulmonary congestion (Fig. 2). Transthoracic echocardiogram was pertinent for left ventricular ejection fraction (LVEF) of 45\%, segmental hypokinetic, eccentric left ventricular hypertrophy, left ventricular dilatation, mild pulmonal regurgitation, and no pericardial effusion. He was diagnosed with late onset STEMI extensive anterior, cardiogenic shock, pulmonary congestion, and new onset complete RBBB. Then, he was admitted to the cardiac intensive care unit.

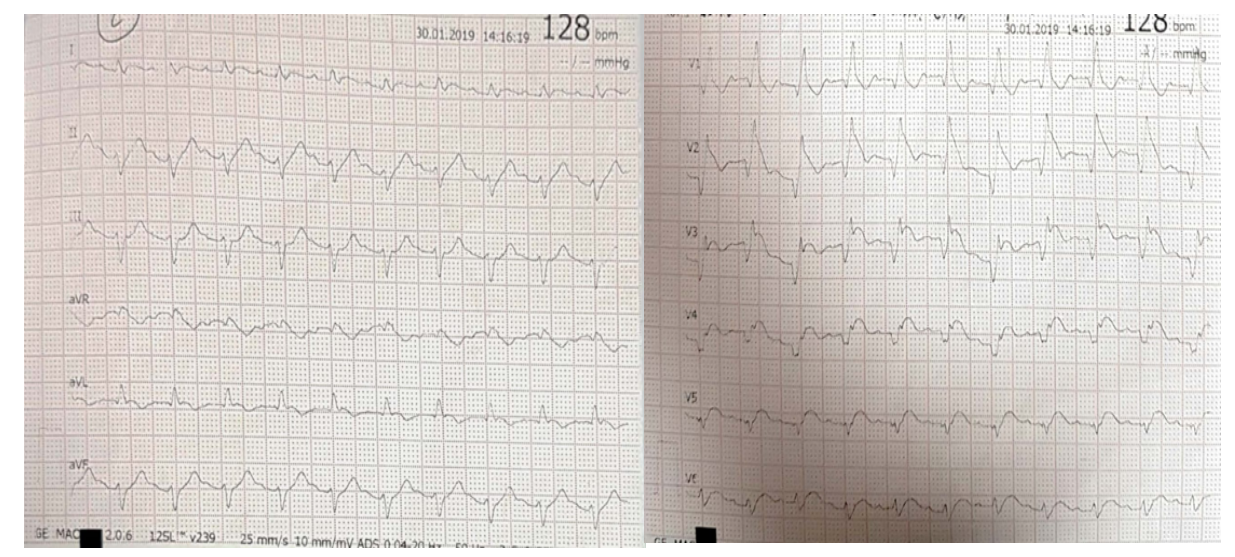

Fig. 1. Electrocardiogram showed ST elevation in lead V1-V6, I, aVL and complete RBBB 


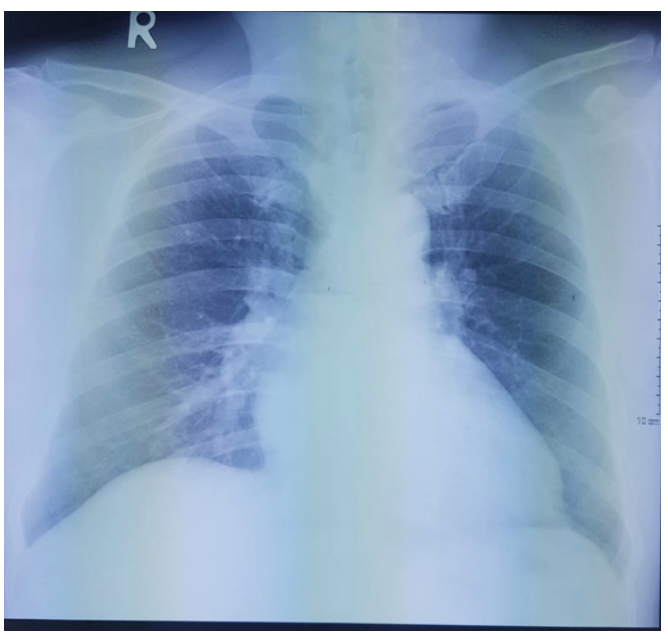

Fig. 2. Chest x-ray showed pulmonary congestion

Medical treatments that were given are inotropic agents, anticoagulant, diuretic, double antiplatelet, and statin. His condition gradually stabilized and chest pain was improved then he was admitted to low care unit and initiated on loop diuretic, mineralocorticoid receptor antagonist (MRA), angiotensin converting enzyme (ACE) inhibitor, digoxin, and ivabradin. Several days after underwent treatment in low care unit, he had no complaint and stable hemodynamic. Repeated ECG was performed before discharged, revealing sinus normal rate with normal frontal axis, counter clockwise rotation, complete RBBB, and deeper pathologic $Q$ and decreased ST elevation in in V1-V6, I, aVL (recent AMI extensive anterior). Transthoracic echocardiogram that was also repeated, showed LVEF of 47\%, segmental hypokinetic, eccentric left ventricular hypertrophy, left ventricular dilatation, mild pulmonal regurgitation, moderate $\mathrm{PE}$ at right lateral, and mild pericardial effusion at posterior and left lateral area of pericardial (Fig. 3). He was discharged with angiography planning being performed electively. Three weeks after discharged, he had no complaints, stable hemodynamic, and the PE was evaluated by transthoracic echocardiogram, showed no pericardial effusion. 


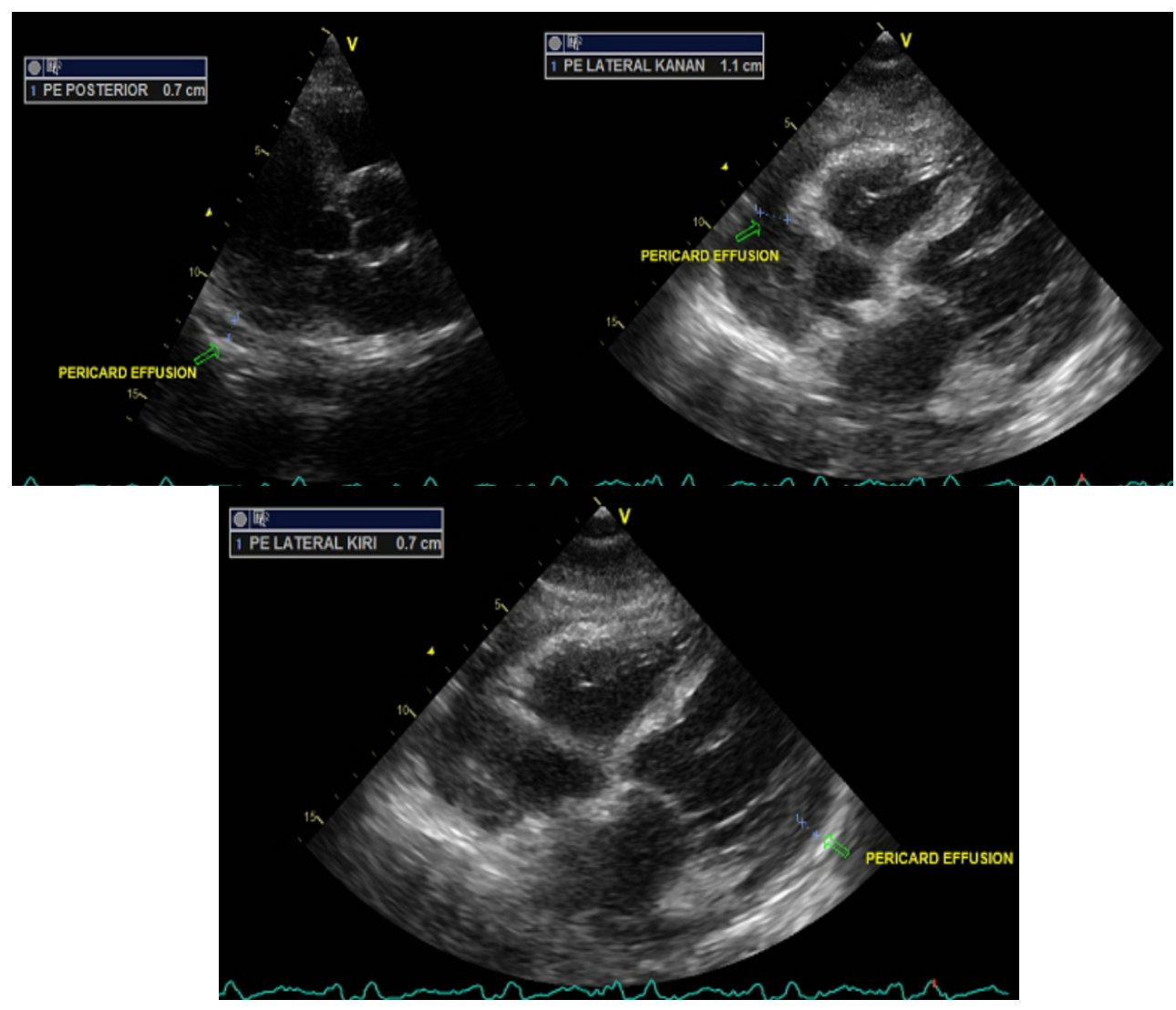

Fig. 3. Echocardiogram showed moderate pericardial effusion (on day $9^{\text {th }}$ )

\section{Discussion}

\subsection{Pathophysiology}

PE is considered to be associated with anterior STEMI, with large infarcts, and when heart failure is present(Bière et al. 2015; Galve et al. 1986; Gueret et al. 2008). Anterior MI is more frequent and so is the PE(Hafiz-Ur-Rehman et al. 2010). This is in accordance with the diagnosis of the patient. The ECG obtained extensive anterior STEMI images and heart failure with cardiogenic shock was found. Rehman et al reported $16.5 \%, 14 \%$ and $15 \%$ with anterior MI, inferior MI and lateral MI, respectively(Hafiz-Ur-Rehman et al. 2010).

Many parameters that may increase vascular permeability have been suggested, such as increase in LV filling pressures, intensity of myocardial injury, or microvessel injury and endothelial dysfunction. Microvascular obstruction (MVO) has, indeed, been associated with higher occurrence of PE or wall rupture(Bière et al. 2015). It(Kloner et al. 1980) showed that the end stage of endothelial lesions was responsible for endothelial rupture and intramyocardial hemorrhage. The latter may dramatically decrease LV wall resistance, thereby increasing shearing stress and local inflammatory response. Finally, systolic wall stress (SWS) may be considered as a preclinical marker of LV loading conditions and, thus, of heart failure(Galve et al. 1986; Widimsky and Gregor 1995). Furthermore, SWS has been accused in LV rupture, 
which may occurs at the edges of the infarcted area(Basso, Rizzo, and Thiene 2010). It is generally accepted that the most frequent cause of moderate pericardial effusion (MPE) in STEMI patient is bleeding from the infarcted wall(Figueras et al. 2002; López-Sendón et al. 1992). In the largest series, pericardiocentesis was hemorrhagic in $75 \%$ of patients(López-Sendón et al. 1992). A study(Figueras et al. 2010) demonstrated a clear association between poor presence of PE and ST-segment resolution and especially with tamponade or EMD. Infarct-related coronary artery that is not completely recanalized, appears to be a pathophysiological link to hemorrhagic PE and threatening rupture that may cause PE(Figueras et al. 2010). The patient was too late in getting reperfusion therapy, so that myocardial rupture could be suspected to be the cause of PE. Although it is often associated with LV free-wall rupture (FWR), it can also be produced by heart failure, pericarditis, or myocardial hemorrhage caused by antiaggregant or anticoagulan therapies(Figueras et al. 2010).

Patients with MPE without initial cardiac tamponade (CT) generally considered cases of sub-acute FWR manifest lower mortality, show a higher mortality than those with small PE, and $8 \%$ die suddenly of a likely FWR(Becker et al. 1999; López-Sendón et al. 1992). It(Varma 2016) suggested that MPE without cardiac tamponade (CT) was myocardial rupture was more self-limited than in patients with MPE and CT. A study(Sugiura et al. 2003) have recently reported that 6 of 13 patients (46\%) who died of FWR had shown an MPE without CT before the fatal event. Unless proved otherwise FWR is the most probable cause of MPE with $\mathrm{CT}$ in the setting of MI, other less common causes include aortic dissection and pericarditis(Varma 2016).

\subsection{Management}

Due to its variable clinical course, early diagnosis of etiology of MPE is important. The most important aspect in the diagnosis of FWR, is to entertain the possibility in the presence of MPE(Varma 2016). A study(Figueras et al. 2010) suggested that clinicians should look for PE early, and if MPE is present, they should suspect that FWR may be present or evolving. The suspicion of FWR is augmented if the PE is hemodynamically compromising, especially if EMD occurs. Common clinical features of sub-acute LV free wall rupture after acute STEMI are: (1) recurrent or persistent chest pain; (2) recurrent or persistent ST segment elevation; (3) hypotension. Integrating these signs into a syndrome can increase the clinician's awareness to the fatal complication(Varma 2016).

A study(Figueras et al. 2010) demonstrated how measurement of hematocrit in pericardial fluid provides important additional insight into the cause of PE. A diagnostic pericardial puncture with aspiration of 5 to 10 $\mathrm{mL}$ of pericardial fluid can usually be done safely with ultrasound guidance. If the hematocrit level is higher than normal value, rupture should be considered the cause. If measurement of hematocrit in the pericardial fluid is performed days later, a decline in hematocrit seems ascribable to the formation of a blood clot and the dilution of the supernatant fluid. The present data also advocate pericardial puncture and drainage to better determine the cause of PE and guide therapy. Thus, pericardial drainage should be considered in patients with moderate PE(Køber et al. 2010).

When the severe prognosis of early to moderate PE is considered, close surveillance and an aggressive therapeutic approach seem warranted. Optimally, the patients should be observed in a cardiac intensive care unit, with close echocardiographic surveillance, in an institution with cardiothoracic surgical backup(Køber et al. 2010). The prognostic value of PE when measured by echocardiography is generally based on examinations made in the first 48 hours rather than at 5 days. On the one hand, infarct size, MVO, and edema should be analyzed after the first 48 hours(Dall'Armellina et al. 2011). In contrast, it(Galve et al. 1986) showed that the PE rate was maximal at day 3 and remained stable until day 10.

In conclusion, the present study of Figueras et al, combined with other available studies, indicates that (1) early echocardiography to detect PE can detect a population with a high risk of death and who deserve close monitoring to detect FWR and, it is hoped, to allow time to react before sudden death; (2) when the PE in a 
parasternal short-axis view exceeds $10 \mathrm{~mm}$, the risk of a FWR is particularly high; and (3) measurement of hematocrit in PE is valuable for detection of FWR(Køber et al. 2010). Also, preliminary observations would suggest that magnetic resonance imaging may help recognize impending FWR(Figueras et al. 2010; Zoni et al. 2003).

Patients with MPE without CT can be initially managed medically(Canovas 2003; López-Sendón et al. 1992). But those with CT have a high mortality, and only a few survive with surgical or medical management. Hence, attempts to significantly reduce mortality clearly require a highly motivated and coordinated medical and surgical team eager to initiate resuscitative maneuvers at any time and to rush the patient to the operating room because these are mostly cases of FWR(Varma 2016). Surgery is indicated in those patients who remained hypotensive or have recurrence of $\mathrm{CT}$, although it is not always performed for a number of reasons (comorbidity, persistence of shock, etc.). In existing series, 30-day mortality with conservative management is not higher than in operated patients(Canovas 2003; López-Sendón et al. 1992).

Its management poses a therapeutic dilemma because not all patients benefit from emergency surgery aimed at treating an underlying FWR and subsequent management may be individualized because a conservative approach is often successful. It is not only size of effusion but also hemodynamic status, cause is important in the management of post MI pericardial effusion. Acute FWR needs immediate surgery. Subacute FWR needs judicious approach with initial medical stabilization followed by surgery. Hence all patients with post MI MPE should be thoroughly evaluated and managed accordingly(Varma 2016).

In this case, when MPE was discovered after being diagnosed with late-onset STEMI, close monitoring of complaints, clinical conditions, optimal medical therapy, and periodic echocardiography examination as described in several recommendations was carried out. However, considering the condition of these patients is getting better, we feel that it is enough with monitoring by non-invasive examination. Fortunately, MPE in these patients with conservative therapy experiences self limited. MRI may be considered in MPE cases if possible to ascertain the cause of PE. Reperfusion therapy for STEMI can be considered later electively.

\section{Conclusions}

PE as a STEMI complication is associated with anterior STEMI, with large infarcts, and presence of heart failure. Entertain the possibility of FWR presence is the most important aspect. In case of heart failure, SWS may be considered as a preclinical marker of LV loading conditions and has been accused in LV rupture, which may occurs at the edges of the infarcted area. When PE is detected, close echocardiographic surveillance, pericardial puncture, and magnetic resonance imaging may be considered to suspect FWR as the cause of PE. Management may be individualized, depends on size of effusion and hemodynamic status, could be conservative or surgery.

\section{Acknowledgements}

Not applicable.

\section{References}

Basso, Cristina, Stefania Rizzo, and Gaetano Thiene. 2010. "The Metamorphosis of Myocardial Infarction Following Coronary Recanalization." Cardiovascular Pathology 19(1):22-28.

Becker, Richard C., Judith S. Hochman, Christopher P. Cannon, Frederick A. Spencer, Steven P. Ball, Michael J. Rizzo, and Elliott M. Antman. 1999. "Fatal Cardiac Rupture among Patients Treated with Thrombolytic Agents and Adjunctive Thrombin Antagonists." Journal of the American College of Cardiology 33(2):479-87.

Bière, Loïc, Victor Mateus, Guillaume Clerfond, Sylvain Grall, Serge Willoteaux, Fabrice Prunier, and Alain Furber. 2015. "Predictive 
Factors of Pericardial Effusion after a First Acute Myocardial Infarction and Successful Reperfusion." American Journal of Cardiology 116(4):497-503.

Canovas, S. J. 2003. "Midterm Clinical and Echocardiographic Results With Patch Glue Repair of Left Ventricular Free Wall Rupture." Circulation 108(90101):237II--240.

Dall'Armellina, Erica, Nina Karia, Alistair C. Lindsay, Theodoros D. Karamitsos, Vanessa Ferreira, Matthew D. Robson, Peter Kellman, Jane M. Francis, Colin Forfar, Bernard D. Prendergast, Adrian P. Banning, Keith M. Channon, Rajesh K. Kharbanda, Stefan Neubauer, and Robin P. Choudhury. 2011. "Dynamic Changes of Edema and Late Gadolinium Enhancement After Acute Myocardial Infarction and Their Relationship to Functional Recovery and Salvage Index.” Circulation: Cardiovascular Imaging 4(3):228-36.

Figueras, Jaume, José A. Barrabés, Vicens Serra, Josefa Cortadellas, Rosa Maria Lidón, Alvaro Carrizo, and David Garcia-Dorado. 2010. "Hospital Outcome of Moderate to Severe Pericardial Effusion Complicating St-Elevation Acute Myocardial Infarction." Circulation 122(19):1902-9.

Figueras, Jaume, Alberto Juncal, Julio Carballo, Josefa Cortadellas, and Jordi Soler Soler. 2002. "Nature and Progression of Pericardial Effusion in Patients with a First Myocardial Infarction: Relationship to Age and Free Wall Rupture." American Heart Journal 144(2):251-58.

Galve, E., H. Garcia-Del-Castillo, A. Evangelista, J. Batlle, G. Permanyer-Miralda, and J. Soler-Soler. 1986. "Pericardial Effusion in the Course of Myocardial Infarction: Incidence, Natural History, and Clinical Relevance." Circulation 73(2):294-99.

Gueret, Pascal, K. Khalife, Y. Jobic, E. Fillipi, K. Isaaz, S. Tassan-Mangina, C. Baixas, P. Motreff, and C. Meune. 2008. "Echocardiography Assessment of the Incidence of Mechanical Complications during the Early Phase of Myocardial Infarction in the Reperfusion Era: A French Multicentre Prospective Registry." Archives of Cardiovascular Diseases 101(1):41-47.

Hafiz-Ur-Rehman, Sher Bahadar Khan, Abdul Hadi, Tariq Nawaz, Syed Tahir Shah, Hameedullah, Adnan Mahmood Gul, and Mohammad Hafizullah. 2010. "Frequency of Pericardial Effusion in Patients with First Myocardial Infarction and Its Effects on In-Hospital Morbidity and Mortality." Journal of Ayub Medical College, Abbottabad: JAMC 22(2):184-86.

Kloner, Robert A., Robert E. Rude, Nancy Carlson, Peter R. Maroko, L. W. DeBoer, and Eugene Braunwald. 1980. "Ultrastructural Evidence of Microvascular Damage and Myocardial Cell Injury after Coronary Artery Occlusion: Which Comes First?" Circulation 62(5):945-52.

Køber, L., J. E. Møller, and C. Torp-Pedersen. 2010. "Moderate Pericardial Effusion Early After Myocardial Infarction." Circulation 122(19):1898-99.

López-Sendón, José, Ana González, Esteban López de Sá, Isabel Coma-Canella, Inmaculada Roldán, Francisco Domínguez, Isidoro Maqueda, and Luis Martín Jadraque. 1992. "Diagnosis of Subacute Ventricular Wall Rupture after Acute Myocardial Infarction: Sensitivity and Specificity of Clinical, Hemodynamic and Echocardiographic Criteria." Journal of the American College of Cardiology 19(6): 1145-53.

Mazzoni, V., A. Taiti, A. Bartoletti, A. Monopoli, N. R. Petix, and A. Bini. 2000. "The Spectrum of Pericardial Effusion in Acute Myocardial Infarction: An Echocardiographic Study." Italian Heart Journal: Official Journal of the Italian Federation of Cardiology 1(1):45-49.

Sugiura, Tetsuro, Yo Nagahama, Seishi Nakamura, Yoshihiro Kudo, Fumiyasu Yamasaki, and Toshiji Iwasaka. 2003. "Left Ventricular Free Wall Rupture after Reperfusion Therapy for Acute Myocardial Infarction.” The American Journal of Cardiology 92(3):28284.

Varma, G. 2016. "Post MI Pericardial Effusion-'Size Does Matter."” Indian Journal of Cardiovascular Disease in Women WINCARS 01(04):013-018.

Widimsky, P. and P. Gregor. 1995. "Pericardial Involvement during the Course of Myocardial Infarction: A Long-Term Clinical and Echocardiographic Study." Chest 108(1):89-93.

Zoni, Antonello, Arianna Arisi, Domenico Corradi, and Diego Ardissino. 2003. "Magnetic Resonance Imaging of Impending Left Ventricular Rupture After Acute Myocardial Infarction.” Circulation 108(4):498-99. 